\title{
The Digital Tumor Images System Design and its Contour Detection Using an Improved Level Set Algorithm
}

\author{
$\mathrm{Na}$ Ying \\ Institute of Communication Engineering \\ Hangzhou Dianzi University \\ Hangzhou, China \\ e-mail: yingnaxj@hdu.edu.cn \\ Qingbiao Yang \\ Institute of Communication Engineering \\ Hangzhou Dianzi University \\ Hangzhou, China \\ e-mail:279481626@qq.com \\ Qinfang Wang \\ Institute of Communication Engineering \\ Hangzhou Dianzi University \\ Hangzhou, China \\ e-mail:1028504704@qq.com
}

\author{
Jianwei Liu \\ Institute of Communication Engineering \\ Hangzhou Dianzi University \\ Hangzhou, China \\ e-mail: name@xyz.com \\ Xuzhen Wang \\ Institute of Communication Engineering \\ Hangzhou Dianzi University \\ Hangzhou, China \\ e-mail: name@xyz.com \\ George Giakos \\ Electrical and Computer Engineering, \\ Manhattan College \\ New York, USA \\ e-mail: giakos@manhattan.edu
}

\begin{abstract}
Because digital diagnostic pathology allows people to acquire, store and analyze pathological information from the images of immunohistochemical glass slides which are scanned to create digital slides, this paper introduced a system design to convert the slides to digital images And it has become one of the most valuable and convenient advancements in technology over the past years. According to the built system, this paper acquired and analyzed the digital pictures from the slides. This analyzed method introduced OSTU segment and an improved fast level set algorithm to outline the tumor contour. Simulations results show that the image analyze algorithm is effective to segment the tumor from the other parts.
\end{abstract}

Keywords:Polarized Light, Brain Cancer Detection, Digital Pathology,Level Set

\section{INTRODUCTION}

Compared with the traditional pathology technology, digital pathology can provide reliability and repeatability. However, pathological using digital platform has been developed slowly and its application has been limited to the education, research and the special clinicalpractice. So the digital pathology needs to do a lot of preparatory work before it waswidely used.

The digitalization of pathology can offer the view and analyze the data and samples from any location in the world[1-3].The use ofthis method of"remotepathology" can translate the image data easily through the Internet and reduce the manual labor in the labs.Pathologydigitizationeliminates the traditionalmanualmicroscope or the limitations and shortcomings of pathology,for example, it can adjustthe quality of image andacquire as much as possible exposure rate. This also makes thepathological splices easily to storage and retrieval.Further,analysis and process them to extract and diagnosis the related informationof theimage, research the tumormorphology and detect diseases, evaluate the organization'sfunction and the metabolicstage.

Therefore,this paper proposed the edge extraction method that after the OSTU of twovalued processing, usethe fast contour segment algorithm based on the level set,so that the non-normal part of the digital images acquired by brainpathologyto be detected rapidly. The algorithm is improved to enhance the convergence speed by thelevel set algorithm on the irregular pictures and get the earlybraintumor contouraccurately, provide an effectivereferenceto thepathologystaffand doctors.

\section{SYSTEM DESIGN}

Because of the particularity of the pathological splices, it need translate system to convert them intodigitalimages[4].

As shown in Fig. 1,the system firstinput the light source,and then through afilter,a polarizer and aphaseretarder, and the beam goes through thesample slide,the other phaseretarder and a polarizer, finally convertthe sample splice into digital image by 
thecameraor otherdetector. This system can put thepathologicalsample slidesinto clear digital images.

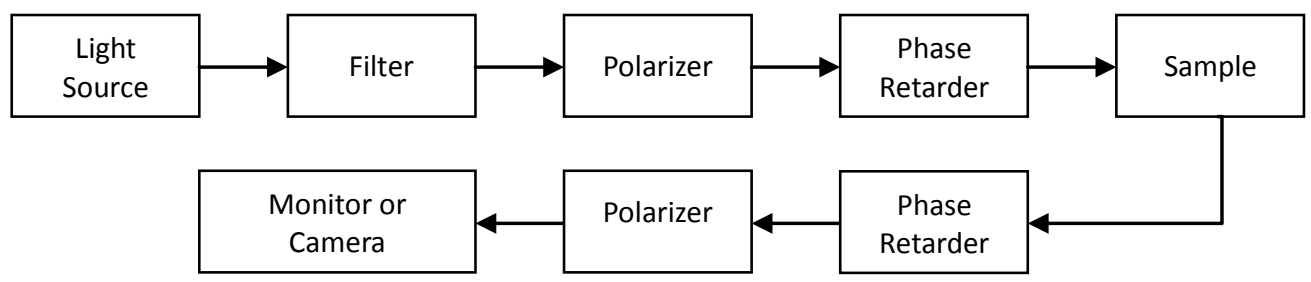

Figure 1. System Design

In the Fig.1, the light source can be white light or laser, such as $785 \mathrm{~nm}, 830 \mathrm{~nm}, 1050 \mathrm{~nm}$. The filtercontains coloredfilters $(488 \mathrm{~nm}, 546 \mathrm{~nm}, 610 \mathrm{~nm}, 750 \mathrm{~nm})$ andother anglesfilter;polarizercan be linear or ring; pathological section can behuman tissues,lung cancer cells,braintumor,plastics,seleniumfilm or other substances;monitoroften uses CMOS camera or a colorcamera.

Based on the basic system, the experiment according to the different objects, light sources, filters, polarization's angle etc.,obtainthe most satisfactorypictures. This paperobtained thedigital images of the pathological splices through the system.

\section{THE AlgORITHM PRINCIPLE}

\section{A. The Basic Principle of the Distance Level SetMethod}

In 1987, Osher and Sethian proposed a method called level set which obtained the object contour and boundary by a group of evolution curves[5]. But the disadvantage is this method need re- initialization in slow speed. And in recent years it was used very broadly [6,7]. Li[8] proposedevolutionarymethod which needn'tre-initialize through adopted a internal energy function, a finite difference methodand a bigger practice step size to solve the corresponding partial differential equations. That algorithm is called distance level setand improves the evolution speed.

The algorithm assuming the initial contourcurve is $\mathrm{C}$ which is represented by the following formula

$\frac{\partial \mathrm{C}(\mathrm{s}, \mathrm{t})}{\partial \mathrm{t}}=\mathrm{FN}$

whereFis the speedfunction controlling the profilechanges, $\mathrm{N}$ is the inside vector function with the same curvature as $\mathrm{C}$; the contourcurve $\mathrm{C}$ is shown as a group ofthe level setfunction of times, and its internaldata is negativeand theexternal datais positive.

Li presented the energyfunction:

$\mathrm{E}(\varphi)=\mu \mathrm{P}(\varphi)+\mathrm{E}_{\mathrm{m}}(\varphi)$
In the first part: $\mu$ is a common values, $P(\varphi)$ is the internal energy, on behalf ofthe level setfunctions deviates from thesign function's distance,as shown in the formula (3):

$$
\mathrm{P}(\varphi)=\int_{\Omega} \mathrm{P}(|\nabla \varphi|) \mathrm{dxdy}
$$

where $\Omega$ is the image area, I (x,y) means images. The second part $\mathrm{E}_{\mathrm{m}}(\varphi)$ is the outside energy function which drives the initial level set curve movetoward the goal,when reaching the targetposition, the value of $\mathrm{E}_{\mathrm{m}}(\varphi)$ iszero:

$$
\mathrm{E}_{\mathrm{m}}(\varphi)=\lambda \operatorname{Lg}(\phi)+\alpha \operatorname{Ag}(\phi)
$$

$=\lambda \int_{\Omega} \mathrm{g}(\nabla \mathrm{I}) \delta(\varphi)|\nabla \varphi| \mathrm{dxdy}+\alpha \int_{\Omega} \mathrm{g}(\nabla \mathrm{I}) \mathrm{H}(-\varphi) \mathrm{dxdy}(4)$ among them, $\lambda>0$ and $\alpha$ is a constant, $g(s)$ is a function of suspend speedfunction, generally, it's inversely proportional to $\mathrm{x}$, andclose tothe edge as soon as possible, andalsocanmonitorthe weak edge. In [8], thefirst step is filtered by the Gaussian kernel and get $\mathrm{s}=\nabla \mathrm{G}_{\sigma} * \mathrm{I}$, then to obtain the

$$
\mathrm{g}(\mathrm{s})=\left(1+\mathrm{s}^{2}\right)^{-1}
$$

$\delta(\mathrm{x}) 、 H(\mathrm{x})$ are theone-dimensional Diracfunction and Heviside function, the same as in manyliterature:

$$
\begin{gathered}
\delta_{\varepsilon}(\mathrm{x})=\left\{\begin{array}{c}
\frac{1}{2 \varepsilon}\left[1+\cos \left(\frac{\pi \mathrm{x}}{\varepsilon}\right)\right], \quad|\mathrm{x}| \leq \varepsilon \\
0, \quad|\mathrm{x}| \geq \varepsilon
\end{array}\right. \\
\mathrm{H} \varepsilon(\mathrm{x})=\left\{\begin{array}{c}
\frac{1}{2}\left(1+\frac{\mathrm{x}}{\varepsilon}+\frac{1}{\pi} \sin \left(\frac{\pi \mathrm{x}}{\varepsilon}\right)\right), \quad|\mathrm{x}| \leq \varepsilon \\
1, \quad|\mathrm{x}|>\varepsilon \\
0, \quad|\mathrm{x}|<-\varepsilon
\end{array}\right.
\end{gathered}
$$

Which $\delta_{\varepsilon}(\mathrm{x})$ is the difference of $\mathrm{H}_{\varepsilon}(\mathrm{x})$, i.e. $\mathrm{H}_{\varepsilon}^{\prime}(\mathrm{x})=$ $\delta_{\varepsilon}(\mathrm{x})$; parameter $\varepsilon=1.5$.

\section{B. The Proposed Algorithm}

In this paper, after obtained the digital image from the designed system asin Fig1, it needsto identify the lesion part toprovide referencetothe doctors.According to the obtained image characteristics:

(1)The image mainly displays in gray feature,so usinggray scale processing method.

(2)There is a lot ofnoise in theimage which most partis caused by theformalin; meanwhile,there are also othercells. Ifdirectlyusing the level set algorithm, it would getthe wrong edgeand the contour maybesunken deeply.

(3) The original algorithm is not fast enough.

So this paperadopts two-step improvedalgorithm based on [8], as shown in Fig 2. 


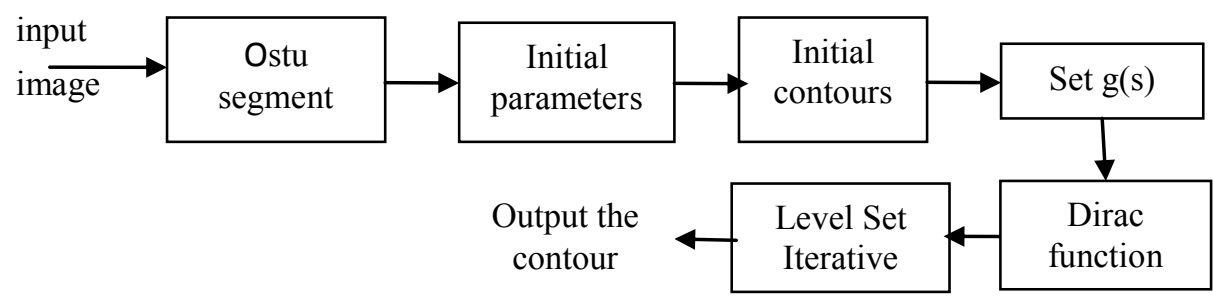

Figure2. The Algorithm Block Diagram

First, use OSTU algorithm to remove unnecessary noise.Then,set thedistancelevel set's parameters; consideringthe speed of the algorithm, this paper use the initial curve outside of the object;Diracfunction takes another formula different with formula (6) and (7).

(1)The initial contourcurve is set in thetarget'soutside and evolutes inside direction. Because of the tumor cells' show deep gray, and the noise gray scale is shallow, the algorithm computes thecenter of the image to reduce the detection area and enhance the evolutionspeed.

(2)After carefulanalysis of the originalalgorithm, the effect of the weight coefficientis very large,because it willchange the amplitude of $g(s)$. After many times experiments, let $\alpha=4$.

According to the $[9,10]$, because the formula (6) is only acting on the local and easy to make thecurve evolution missed into a local minimum, but lost the contours of objects and can't get the boundary of deep sunkenregion andmulti objects, this paper using the regularized DIRACfunction instead of formula (6)

$$
\delta_{\varepsilon}(\mathrm{x})=\frac{1}{\pi} \frac{\varepsilon}{\varepsilon^{2}+\mathrm{x}^{2}}, \lim _{\varepsilon \rightarrow 0} \delta_{\varepsilon}(\mathrm{x})=\delta(\mathrm{x})
$$

After the abovetreatment, the iterations number may reduce much and it still get the satisfied contour.

\section{THE EXPERIMENTAL RESULTS}

The experimentalobject is get from the second part of this paper. And the digital imagesacquired froma brain tumorin the slides which are from a hospital. Because the images are similar, here show the sample and its results.

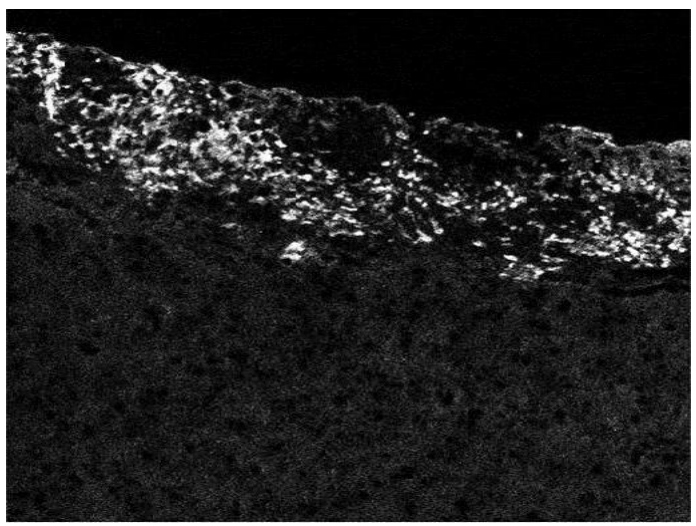

(a)The digital image from the system

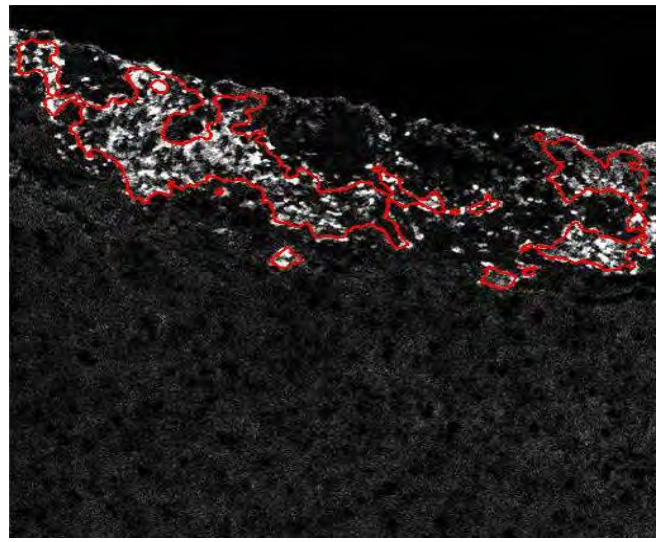

(b)images after OSTU

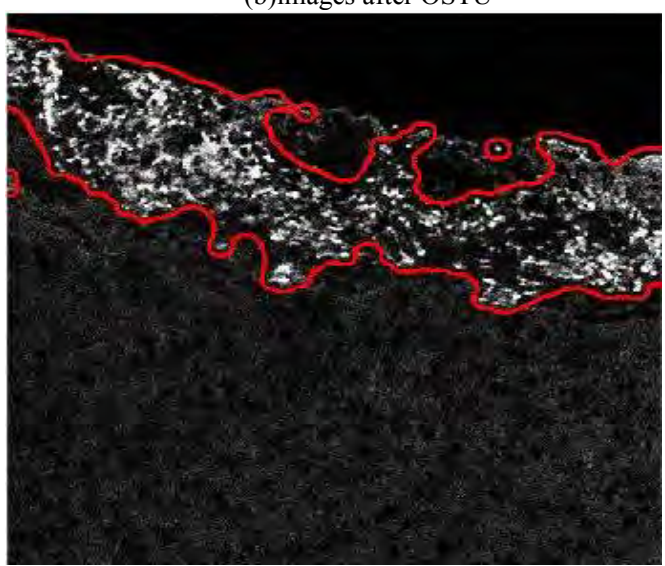

(c) Li's algorithm's result

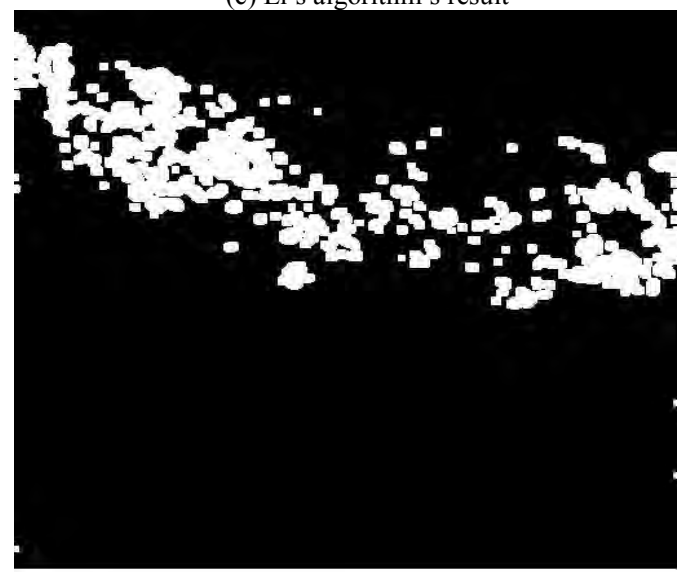

(d) the proposed algorithm's result

Figure 3. the simulate images 
Fig. 3 (a) is the digital image from the tumor slides acquired by the built system. Fig. 3(b)is the result after OSTU segment. Fig.3 (c) is the result of the Li's algorithm; (d) is the result of the proposed algorithm.

In the view of the Fig. 3, (a) is the image getsthrough the built system; (b) is the image after OSTU segment method. It shows that delete the noise clearly; (c) is the result of the Li's algorithm with the original image. And its result lost many useful tumor points and can't obtain the correct edge. Also, it can't be used after the OSTU method because the edge is disappeared. it cost 510 time's iterative. And Fig. 3(d) is the result of the proposed algorithm which can delete the most noise and get the accurate contour. Meanwhile, it cost only 80 times iterative. So the proposed algorithm is proved to be effective to the tumor images.

\section{CONCLUTION}

In this paper, through the establishment of the system, the pathological slides are converted to digital images, which is the research trend of the discipline of pathology. And in the early tumor treatment and medical research, it has the vital significance.

The proposed algorithm in this paper gets good results: 1) this algorithm is faster, and also can extract the good contour; 2) it can solve the problem that the contour stop at the aim position, not deep in depression boundary; 3) avoid the interference of much noise points.

\section{ACKNOWLEDGMENT}

The Project Supported by Zhejiang Provincial Natural Science Foundation of China (LY12F01009); Research on
Application of technology for public welfare of Zhejiang Province (2014C31056).

\section{REFERENCES}

[1] G. C. Giakos,etc. "Polarimetric wavelet phenomenology of space materials". Imaging Systems and Techniques (IST), 2011 IEEE International Conference on 17-18 May 2011 pp:1-6.

[2] G. C. Giakos,C. Narayan, etc. "Polarimetric phenomenology of photons with lung cancer tissue". Measurement Science \& Technology - MEAS SCI TECHNOL, vol. 22, no. 11, 2011.

[3] Justin S. Baba. "Development and calibration of an automated Muellermatrix polarization imaging system" Journal of Biomedical Optics 7(3),pp: 341-349,July 2002

[4] Nirmalya Ghosh. "Tissue polarimetry: concepts, challenges applications, and outlook". Journal of Biomedical Optics 16(11), 110801 (November 2011)

[5] Osher, J. Sethian. "Fronts propagating with curvature-dependent speed: Algorithms based on Hamilton-Jacobi formulations,"J.Comput. Phys., vol. 79, no. 1, pp. 12-49, Nov. 1988.

[6] JamesA.Sethian.LevelSetMethodsandFastMarchingMethods.Camb ridgeUniversityPress(1999).

[7] Qian Yun, Zhang Yingjie. "Level Set Methods and Its Application on Image Segement." Journal of Image and Graphics. Vol 13, No 1, Jan ,2008.

[8] Changcai Yang."Level Set Based Image Segmentation Method", 2008. China Three Gorges University

[9] Chunming Li, etc. "Distance Regularized Level Set Evolution and ItsApplication to Image Segmentation" IEEE TRANSACTIONS ON IMAGE PROCESSING, VOL. 19, NO. 12, DECEMBER 2010,pp:3243-3254.

[10] HE Chuan-Jiang, etc. "Adaptive Distance Preserving Level Set Evolution for Image Segmentation"Journal of Software, Vol.19, No.12, December 2008, pp.3161-3169 